\title{
Kidney Cancer Research Network of Canada (KCRNC) consensus statement on the role of adjuvant therapy after nephrectomy for high-risk, non-metastatic renal cell carcinoma: A comprehensive analysis of the literature and meta-analysis of randomized controlled trials
}

Pierre I. Karakiewicz, MD, FRCSC, MPH ${ }^{1}$; Emanuele Zaffuto ${ }^{1,2}$; Anil Kapoor, MD, FRCSC ${ }^{3}$; Naveen S. Basappa, MD, FRCPC ${ }^{4}$; Georg A. Bjarnason, MD, FRCPC ${ }^{5}$ Normand Blais, MD ${ }^{6}$, MSc; Rodney H. Breau, MD, FRCSC, MSc ${ }^{7}$; Christina Canil, MD, FRCPC ${ }^{8}$; Darrel Drachenberg, MD FRCSC ${ }^{9}$; Sebastien J. Hotte, MD, FRCPC, MSc ${ }^{10}$; Claudio Jeldres, MD, MSc, FRCPC $^{11}$; Michael A.S. Jewett, MD, FRCSC ${ }^{12}$; Wassim Kassouf, MD, CM, FRCSC ${ }^{13}$, Christian Kollmannsberger, MD, FRCPC ${ }^{14}$; Luke T. Lavallée, MDCM, MSc, FRCSC ${ }^{7}$, Ranjena Maloni, BSc, CCRP ${ }^{12}$; Francois Patenaude, MD, FRCPC ${ }^{15}$; Frédéric Pouliot, MD PhD ${ }^{16}$; M. Neil Reaume, MD, FRCPC, MSc ${ }^{8}$; Robert Sabbagh, MD, FRCSC, MSc ${ }^{11}$; Bobby Shayegan, MD, FRCSC $^{3}$; Alan So, MD, FRCSC ${ }^{17}$; Denis Soulières, MD, FRCPC ${ }^{18}$; Simon Tanguay, MD, FRCSC $^{13}$; Lori Wood, MD, FRCPC ${ }^{19}$; Marco Bandini ${ }^{1,2}$

${ }^{1}$ Cancer Prognostics and Health Outcomes Unit, University of Montreal Health Centre, Montreal, QC, Canada; ${ }^{2}$ Division of Oncology/Unit of Urology, URI, IRCCS Ospedale San Raffaele, and Vita-Salute San Raffaele University, Milan, Italy; ${ }^{3}$ Division of Urology, McMaster University, Hamilton, ON, Canada; ${ }^{4}$ Department of Oncology, University of Alberta, Cross Cancer Institute, Edmonton, AB, Canada; ${ }^{5}$ Division of Medical Oncology/Hematology, Sunnybrook Odette Cancer Centre, University of Toronto, Toronto, ON, Canada; ${ }^{6}$ Division of Medical Oncology/Hematology, Centre Hospitalier de l’Université de Montréal, Montreal, QC, Canada; ${ }^{7}$ Clinical Epidemiology Program and Division of Urology, The Ottawa Hospital Research Institute and University of Ottawa Ottawa, ON, Canada; ${ }^{8}$ Division of Medical Oncology, The Ottawa Hospital Cancer Centre and the University of Ottawa, Ottawa, ON, Canada; ${ }^{9}$ Section of Urology, University of Manitoba, Winnipeg, MB, Canada; ${ }^{10}$ Juravinski Cancer and McMaster University, Hamilton, ON, Canada; ${ }^{11}$ Centre hospitalier de l’Université de Sherbrooke, Sherbrooke, QC, Canada; ${ }^{12}$ Departments of Surgery (Urology) and Surgical Oncology, Princess Margaret Cancer Centre, University Health Network, Toronto, ON, Canada; ${ }^{13}$ Division of Urology, McGill University, Montreal, QC, Canada; ${ }^{14}$ Division of Medical Oncology, British Columbia Cancer Agency-Vancouver Cancer Centre, and the University of British Columbia, Vancouver, BC, Canada; ${ }^{15}$ Department of Medicine, Hematology Service and Department of Oncology, Sir Mortimer B. Davis Jewish General Hospital and McGill University, Montreal, QC, Canada; ${ }^{16}$ Division of Urology, Department of Surgery, Université Laval, Quebec, QC, Canada; ${ }^{17}$ Department of Urologic Sciences, The University of British Columbia, Vancouver, BC, Canada; ${ }^{18}$ Division of Medical Oncology/ Hematology Centre Hospitalier de l'Université de Montréal, Montreal, QC, Canada; ${ }^{19}$ Department of Medicine and Urology, Dalhousie University, Halifax, NS, Canada

Cite as: Can Urol Assoc J 2018 Mar. 1; Epub ahead of print. http://dx.doi.org/10.5489/cuaj.5187

Published online March 1, 2018 


\begin{abstract}
Introduction: The Kidney Cancer Research Network of Canada (KCRNC) collaborated to prepare this consensus statement about the use of target agents as adjuvant therapy in patients with non-metastatic renal cell carcinoma (nmRCC) after nephrectomy. We reviewed the published data and performed a meta-analysis of studies that focused on vascular endothelial growth factor receptor (VEGFR) tyrosine kinase inhibitors (TKIs).

Methods: A systematic literature search identified seven trials on adjuvant target therapy in nmRCC. Three trials, the ASSURE, S-TRAC, and PROTECT, focused on VEGFR TKIs and represented the focus of the study, including a meta-analysis combining their data on disease-free survival (DFS) and overall survival (OS).

Results: The ASSURE trial showed no DFS or OS benefit of TKIs over placebo after one year of adjuvant sorafenib or sunitinib. In contrast, the S-TRAC trial showed improved DFS after one year of adjuvant sunitinib using central review process, but not using investigator review process. No OS benefit was recorded in either study. Recently, the PROTECT trial also showed no DFS or OS benefit when one year of adjuvant pazopanib was compared to placebo. Meta-analyses of the pooled DFS and OS estimates from all three trials resulted in DFS and OS hazard ratios of 0.87 (95\% confidence interval [CI] 0.73-1.04) and 1.04 (95\% CI 0.89-1.22), respectively. Conclusions: Data from three available clinical trials of adjuvant VEGFR TKIs vs. placebo do not currently support the use of adjuvant TKI therapy as standard of care after nephrectomy for nmRCC. At this time, adjuvant TKI-based adjuvant therapy is not recommended for routine use after nephrectomy for high-risk nmRCC, but highly motivated patients may benefit from a discussion with their oncologist regarding the risks and benefits of adjuvant TKI.
\end{abstract}




\section{Introduction}

Several trials were designed to evaluate the effect of adjuvant therapy in patients with nonmetastatic renal cell carcinoma (nmRCC), treated with either partial or radical nephrectomy. In this report, we review the current evidence regarding adjuvant targeted agent therapy after nephrectomy for nmRCC and provide a meta-analysis of the three published vascular endothelial growth factor receptor (VEGFR) tyrosine kinase inhibitors (TKIs)-based adjuvant trials [1-3], as discussed at the 2017 Canadian Kidney Cancer Forum (CKCF)[5] and endorsed by the Kidney Cancer Research Network of Canada (KCRNC)[4]

\section{Evidence acquisition}

\section{Eligibility criteria}

A review of the literature was performed in November 2017 to identify relevant randomized controlled studies evaluating the effect of adjuvant therapy in surgically treated nmRCC using Pubmed, Embase, Medline, and Cochrane library, as well as ClinicalTrials.gov registry. The following key words were used alone or in combination: renal cell carcinoma, adjuvant therapy, antiangiogenic therapy TKI, nephrectomy, target agents, treatments and prognosis. Only English language original articles were considered.

\section{Study selection}

The search yielded seven prospective randomized trials of adjuvant targeted agents after nephrectomy for patients with high-risk nmRCC: ASSURE[1], S-TRAC[2], PROTECT[3], SORCE[6], EVEREST[7], ATLAS[8] and ARISER[9] (Table 1). The ARISER trial examined a carbonic anhydrase IX inhibitor (girentuximab). It reported negative findings and was not considered in this review due to its different mechanism of action and treatment molecule unavailability in Canada. The EVEREST trial focused at mammalian target of rapamycin (mTOR) inhibitor (everolimus) and was not included due to lack of reported findings: Of five trials that focused on adjuvant TKI therapy, three published their findings: ASSURE (sorafenib and sunitinib), S-TRAC (sunitinib) and PROTECT (pazopanib) and represent the focus of this report and of the meta-analysis.

Statistical analyses

A quantitative synthesis (ie, meta-analysis) was performed on the ASSURE, S-TRAC and PROTECT trials. For disease-free survival (DFS) and overall survival (OS) data, hazard ratios (HR) and 95\% confidence intervals (CI) obtained directly from studies were pooled to compare results. 


\section{Evidence synthesis}

The ASSURE trial

The ASSURE trial[1] randomized 1,943 patients with completely resected stage pT1b or greater nmRCC. Patients were randomly assigned (1:1:1) to initially receive 54 weeks of sunitinib ( $n=647$ ) 50 mg once per day orally throughout the first 4 weeks of each 6-week (4 weeks on/2 weeks off) cycle continuous, sorafenib ( $n=649) 400 \mathrm{mg}$ twice per day orally, or placebo ( $\mathrm{n}=647)$. Treatment was discontinued due to toxicity by 193 [44\%] of 438 patients on sunitinib, 199 [45\%] of 441 patients on sorafenib and 47 [11\%] of 444 patients on placebo. Upon review of this data, the starting dose of each drug was subsequently reduced and then individually titrated up to the original full doses if possible. The most common grade 3 or worse adverse events were hypertension (17\% sunitinib patients and 16\% sorafenib patients), hand-foot syndrome (15\% sunitinib patients and 33\% sorafenib patients), rash (2\% sunitinib patients and $15 \%$ sorafenib patients), and fatigue (18\% sunitinib patients and 7\% sorafenib patients). The primary analysis showed no significant differences in DFS between study arms: median of 5.8 years (interquartile range [IQR] 1.6-8.2) for sunitinib (HR: 1.02, 95\% CI: 0.85-1.23, p = 0.8), 6.1 years (IQR 1.7not estimable [NE]) for sorafenib (HR: 0.97; 95\% CI: 0.80-1.17; p = 0.7184), and 6.6 years (IQR 1.5-NE) for placebo. In post-hoc subgroup analyses of pathological stage T3 or T4 patients, American Joint Committee on Cancer (AJCC) stage III-IV patients, as well as patients with Fuhrman grade 3 or 4, sunitinib adjuvant therapy failed to demonstrate either DFS or OS benefit compared to placebo. There were six deaths related to treatment: 4 in sunitinib group vs. 1 in sorafenib group and 1 in placebo group. The ASSURE trial investigators concluded that adjuvant treatment with the VEGFR TKI sorafenib or sunitinib did not improve survival relative to placebo. Furthermore, substantial treatment discontinuation occurred in the treatment arm because of excessive toxicity, despite dose reduction strategies. These results provided an important rationale against the use of these drugs for high-risk nmRCC patients in the adjuvant setting after nephrectomy for nmRCC.

The S-TRAC trial

The S-TRAC trial[2] randomized 615 patients with loco-regional, high-risk clear-cell nmRCC to receive either sunitinib (50 mg per day) or placebo on a 4-weeks-on, 2-weeks-off schedule for 1 year or until disease recurrence, unacceptable toxicity, or consent withdrawal. The primary end point of the study was DFS. Using central radiological review, the median duration of DFS was 6.8 years (95\% CI, 5.8 to not reached) in the sunitinib group and 5.6 years (95\% CI: 3.8-6.6) in the placebo group (HR: 0.76; 95\% CI: 0.59-0.98; $\mathrm{p}=0.03$ ). The statistical significance was not confirmed using investigator radiological review (HR: 0.81; 95\% CI: 0.64-1.02; $\mathrm{p}=0.08$ ). Overall survival (OS) data were not mature at data cut-off. Nonetheless, the reported HR for OS failed to reveal an improvement: 1.01 (95\% CI: 0.72-1.44; $\mathrm{p}=0.94)$.

As in the ASSURE trial, S-TRAC required dose reductions. Adverse events were more frequent 
with sunitinib than placebo (34.3 vs. $2 \%$ ), as were dose interruptions (46.4 vs. $13.2 \%$ ) and discontinuations ( 28.1 vs. $5.6 \%$ ). Grade 3 or 4 adverse events were more frequent in the sunitinib group (48.4\% for grade 3 events and $12.1 \%$ for grade 4 events) than in the placebo group (15.8\% and $3.6 \%$, respectively). The incidence of serious adverse events was similar in both groups (21.9\% for sunitinib vs. $17.1 \%$ for placebo) and no deaths were attributed to toxicity. Contrary to the ASSURE trial, these observations indicated that among patients with high-risk clear-cell nmRCC after nephrectomy, the median DFS duration was significantly longer in the sunitinib group than in the placebo group: 6.8 years (95\% CI, 5.8 to not reached) vs. 5.6 years (95\% CI, 3.8 to 6.6$)$.

\section{The PROTECT trial}

Results from the randomized, double-blind phase III PROTECT trial were recently published. It assessed the effect of pazopanib 800mg vs. placebo in high-risk nmRCC after nephrectomy. It is noteworthy that one year after study initiation, the primary objective (DFS for pazopanib 800mg) was amended based on a high treatment discontinuation rate secondary to adverse events. The primary study objective became DFS for pazopanib $600 \mathrm{mg}$ vs. placebo. Secondary objectives were DFS for pazopanib $800 \mathrm{mg}$ vs. placebo, as well as DFS for pazopanib at either $800 \mathrm{mg}$ or $600 \mathrm{mg}$ vs. placebo. The PROTECT trial also examined OS for both treatment doses, relative to placebo. Overall, the PROTECT trial enrolled 1,538 patients: 571 received pazopanib 600mg, 198 received pazopanib 800mg and 769 received placebo. The study did not meet its primary end-point in the intention-to-treat (ITT) pazopanib 600mg group, as evidenced by DFS HR of 0.86 (95\% CI: 0.70-1.06) relative to placebo. Conversely, the results of the secondary end-point analyses demonstrated a DFS benefit in the ITT pazopanib 800mg group (HR: 0.69; 95\% CI: $0.51-0.94$ ) and in the combined ITT pazopanib 800mg and ITT pazopanib 600mg groups (HR: 0.80; 95\% CI: 0.68-0.95) relative to placebo. The DFS results for the pazopanib $800 \mathrm{mg}$ and the pazopanib $600 \mathrm{mg}$ were conflicting. The OS results are not yet mature and for now are inconclusive. To date, the reported HR for OS failed to reveal an improvement either for pazopanib 600mg (HR: 0.79; 95\% CI: 0.57-1.09; $\mathrm{p}=0.16$ ) or for pazopanib 800mg (HR: 0.9; 95\% CI: 0.55-1.46; $\mathrm{p}=0.66$ ). The final data cut-off for OS analyses is planned for April 2019. Reported adverse event data showed that nearly all (558/568, 98\%) 600mg pazopanib patients and $(501 / 558,90 \%)$ placebo patients experienced treatment-related adverse events. Of those in the ITT $600 \mathrm{mg}$ pazopanib group, $60 \%$ experienced greater than grade $3 / 4$ adverse events vs. $21 \%$ in the placebo arm. Taken together, pazopanib showed no OS benefit, but improved DFS at the $800 \mathrm{mg}$ doses. On the other hand, pazopanib $800 \mathrm{mg}$ and pazopanib $600 \mathrm{mg}$ also contributed to an elevated proportion of side effects.

\section{Meta-analysis}

To examine the combined findings of all three VEGFR TKI-based adjuvant studies including the most recent PROTECT trial, we performed a meta-analysis of the S-TRAC, ASSURE and 
PROTECT results using DFS and OS data from their original reports.[1-3] Median DFS estimates for sunitinib vs. placebo group were 81.6 vs. 67.2 months (HR: 0.76; 95\% CI: 0.590.98; $\mathrm{p}=0.03$ ) in the S-TRAC and 70 vs. 79.6 months (HR: 1.02; 95\% CI: 0.85-1.23; $\mathrm{p}=0.7$ ) in the ASSURE trial. Median DFS estimate for sorafenib vs. placebo was 73.4 vs. 79.6 months (HR: 0.97; 95\% CI: 0.80-1.17; $\mathrm{p}=0.7$ ) in the ASSURE trial. Median recurrence free survival data were not mature in the PROTECT trial. Nonetheless, after 3 years of follow-up, the DFS rates for pazopanib 800mg vs. placebo were respectively 66 vs. 56\% (HR: 0.69; 95\% CI: 0.51-0.94; p = 0.02 ) and 67 vs. 64\% (HR: 0.86; 95\% CI: 0.69-1.06; $\mathrm{p}=0.16$ ) for pazopanib 600mg vs. placebo. The pooled DFS estimates derived from S-TRAC, ASSURE and PROTECT (800mg) resulted in a HR of 0.87 (95\% CI: 0.73-1.04; Figure 1). The pooled DFS estimates derived from the STRAC, ASSURE and PROTECT (600mg) resulted in a HR of 0.92 (95\% CI: 0.82-1.03; Figure 2). Regarding OS outcomes, no study had mature OS data and none reported a statistically significant OS benefit favouring TKI-based treatment. OS rates for sunitinib vs. placebo were 79.3 vs. $79.1 \%$ (HR: 1.01; 95\% CI: 0.71-1.44) in the S-TRAC and 77.9 vs. $80.3 \%$ (HR:1.17; 95\% CI: $0.90-1.52 ; \mathrm{p}=0.17$ ) in the ASSURE trial. OS rates for sorafenib vs. placebo were 80.5 vs. 80.3\% (HR: 0.98; 95\% CI: 0.75-1.28; $\mathrm{p}=0.85$ ) in the ASSURE trial. The reported OS rate for pazopanib 800mg vs. placebo after 3 years of follow-up were 85.4 vs. 82.9\% (HR: 0.90; 95\% CI: 0.55-1.46; $\mathrm{p}=0.66$ ) and 88.6 vs. 85.3\% (HR: 0.79; 95\% CI: 0.57-1.09; $\mathrm{p}=0.16$ ) for pazopanib $600 \mathrm{mg}$ vs. placebo, in the PROTECT trial. The pooled OS estimates derived from S-TRAC, ASSURE and PROTECT (800mg) trials resulted in a HR of 1.04 (95\% CI: 0.89-1.22; Figure 3). The pooled OS estimates derived from S-TRAC, ASSURE and PROTECT (600mg) trials resulted in a HR of 0.99 (95\% CI: 0.85-1.17; Figure 4). Taken together, the meta-analysis results failed to show any statistically significant DFS or OS benefit of adjuvant VEGFR TKI therapy in patients with high-risk nmRCC, when all three trials were considered.

\section{Discussion}

The standard of care for nmRCC remains nephrectomy. However, despite complete resection and negative margins, many patients may experience disease recurrence. The latter puts them at risk of death from RCC.[10] Seven randomized studies attempted to evaluate if adjuvant therapy after nephrectomy improves survival. Of those, five examined the use of VEGFR TKI-based therapy. To date, the ASSURE, S-TRAC and the PROTECT trials have reported their findings. All three studies agreed that toxicity is an important barrier to adjuvant therapy. However, their results regarding the protective effect of adjuvant therapy on DFS conflicted. Several comments regarding these findings deserve mention.

First, ASSURE examined sorafenib and sunitinib in the adjuvant setting after nephrectomy for nmRCC and both agents failed to demonstrate a DFS or OS benefit relative to placebo[1]. The S-TRAC trial showed DFS benefit, without OS benefit[2]. The PROTECT trial failed to show DFS benefit, except for pazopanib 800mg subgroup, where accrual was interrupted due to adverse effects[3]. In all three reports, data have not reached adequate maturity for final 
OS analyses. Nonetheless, not even a trend towards OS benefit from adjuvant therapy was observed in any of three studies. Moreover, lack of meaningful trend toward OS benefit questions the reversal of OS findings with longer follow-up.

Second, DFS data from the ASSURE and S-TRAC trials regarding sunitinib are conflicting. The ASSURE trial showed no DFS benefit in patients treated with sunitinib relative to placebo. Moreover, post-hoc subgroup analyses also failed to show statistically significant DFS benefit in subgroups of patients with more aggressive nmRCC characteristics: pT3-4 (824 patients on sunitinib or placebo), AJCC stages III-IV, as well as in Fuhrman grade 3 and 4 (847 patients on sunitinib or placebo)[1]. These observations suggest that these specific tumor characteristics are not the driver of the null DFS effect observed in the ASSURE trial. Therefore, a study design that would exclusively rely on patients with the most aggressive pathological characteristics would unlikely contribute to greater DFS benefit than that reported. In view of these findings, the S-TRAC trial findings that showed a DFS benefit in sunitinib-exposed patients cannot be solely explained by inclusion of patients with more aggressive disease characteristics. Moreover, it is of interest that within the S-TRAC trial, patients with more aggressive disease characteristics failed to demonstrate a stronger DFS benefit (HR 0.74, investigator review) than that observed in all patients (HR 0.76, investigator review). Thus, both trials suggest that inclusion of patients with more aggressive disease characteristics will result in little, if any additional effect on DFS.

Third, important sunitinib dosing characteristics distinguish both studies and may represent the most plausible explanation for the discordant findings regarding the effect on DFS that were recorded in the ASSURE and the S-TRAC trials. In the ASSURE trial, important dose reductions were required due to treatment toxicity that translated into a $44 \%$ sunitinib discontinuation rate vs. $28 \%$ in the S-TRAC trial. Despite this, in ASSURE, a median of eight six-week sunitinib cycles (of an expected total of 9 cycles) were administered to those who completed treatment (56\%). Moreover, the proportion of sunitinib patients, who received the intended ASSURE sunitinib dose at cycle three was only $42 \%$. However, at mid study, the starting dose reduction threshold was reduced from 50 to $37.5 \mathrm{mg}$, to avoid elevated treatment discontinuation rate and this starting dose was administered to 34\% of ASSURE patients. In addition, dose reductions to $25 \mathrm{mg}$ were allowed in ASSURE. In post-hoc ASSURE analyses, comparisons of patients who started sunitinib at a reduced dose vs. placebo patients, favoured the placebo arm. In consequence, the ASSURE trial data suggest that dose reductions contributed to inferior response rates. Sunitinib dosing also warrants close examination within the S-TRAC trial. Here, the starting dose was $50 \mathrm{mg}$ and dose reductions down to 37.5 , but not to $25 \mathrm{mg}$, were allowed. This observation may at least in part explain better DFS benefit of sunitinib in S-TRAC, relative to that reported in the ASSURE trial. In consequence, dose escalations such as described by Bjarnason et al.[11,12], might result in better DFS and possibly also in better OS and dose individualization may warrant consideration, if adjuvant sunitinib therapy is considered. 
Fourth, additional differences between study designs of the ASSURE and S-TRAC regarding sunitinib need to be considered. Foremost of these is the sample size difference that distinguishes ASSURE from S-TRAC. Specifically, the ASSURE trial randomized 647 patients to the sunitinib and placebo arms each. The S-TRAC trial was smaller with only 309 and 306 patients randomized to sunitinib or placebo, respectively. The sample size differences invariably weigh on the results of all meta-analyses. Indeed, the European Association of Urology (EAU) RCC guideline panel commented on a meta-analysis[13] of the ASSURE and S-TRAC trials, to help reconcile the conflicting DFS data. The pooled ASSURE and S-TRAC derived DFS and OS estimates showed no differences favouring sunitinib over placebo: pooled DFS estimates resulted in a HR of 0.89 (95\% CI: 0.67-1.19) and pooled OS estimates resulted in a HR of 1.12 (95\% CI: 0.92-1.35). The meta-analysis exclusively focused on the sunitinib data of the ASSURE and on the central review for the S-TRAC trial. It is also important to note that the negative ASSURE trial had no central review. Conversely, the positive DFS findings were reported upon central review of the S-TRAC data. Similar to ASSURE, investigator review within the S-TRAC trial showed no statistically significant DFS benefit of adjuvant sunitinib, when all patients were analyzed (HR $0.8195 \%$ CI 0.64-1.02), as well as when only patients with more aggressive characteristics were analyzed (HR 0.76 95\% CI 0.58-1.01). This observation has two implications. Firstly, it shows that central review would have been ideal within the ASSURE study. It is however unlikely that central review would have changed the reported DFS from truly insignificant (HR 1.02, 95\% CI: 0.85-1.23; $\mathrm{p}=0.8$ ) to statistically significant and clinically relevant findings. Secondly, it is important to note the effect of central vs. investigator review on reported DFS in all patients, as well in higher-risk sub-group in the S-TRAC study. In both STRAC analyses, central review yielded statistically significant results (all patients HR: 0.76 [95\% CI: 0.59-0.98)]; higher-risk patients HR: 0.74 [95\% CI: 0.55-0.99]), while investigator review resulted in loss of statistical significance (all patients HR: 0.81 [95\% CI: 0.64-1.02]; high-risk patients HR: 0.76 [95\% CI: 0.58-1.01]). Such sensitivity to radiological interpretation (central vs. investigator review) questions the robustness of the S-TRAC trial findings especially given that central review is not feasible in the real world clinical environment.

Fifth, it is of note that a third prospective randomized controlled trial of a VEGFR inhibitor, the PROTECT trial that relied on pazopanib, also failed to demonstrate an OS benefit[1-3]. However, its results regarding DFS are equivocal. Specifically, the comparison between pazopanib $800 \mathrm{mg}$ vs. placebo revealed a statistically significant DFS benefit. However, study design modifications towards pazopanib 600mg dosing scheme did not result in a statistically significant DFS benefit. Differences in follow-up length might explain the difference: 800mg pazopanib patients were treated in the early part of the study and those exposed to 600 $\mathrm{mg}$ were treated in the later part, with resulting shorter follow-up at data cut-off. Moreover, higher dose intensity of 800mg might also add to DFS benefit. Taken together, the contribution of the 
PROTECT trial corroborates the lack of OS benefit and also does not modify the body of evidence regarding DFS benefit. To integrate the findings of the PROTECT trial to those of the ASSURE and S-TRAC trials, we performed a meta-analysis regarding the pooled three trial DFS and OS. Lack of DFS benefit in the pooled analysis was obtained, even when using the best casescenario for pazopanib data, namely those recorded with $800 \mathrm{mg}$ dosing. Lastly, it is of interest to examine opinions of expert clinicians, such as the EAU RCC panellists, regarding their perception of the ASSURE and S-TRAC meta-analysis findings[14]. Here, review of several hypothetical scenarios revealed that the beneficial effects of adjuvant therapy on DFS is not sufficient to be considered as practice changing. Conversely, a protective effect on OS, quantified with a HR of 0.75 or better could represent a practice-changing finding. This observation implies that even statistically significant meta-analysis derived DFS benefits would not be a sufficient substitute for OS benefit.

\section{Conclusions}

To date, RCTs of adjuvant therapy after nephrectomy for nmRCC showed no OS benefit and equivocal DFS benefit. These findings are confirmed by our meta-analysis of three RCTs of adjuvant VEGFR TKI therapy for nmRCC. In consequence, it is the opinion of the KCRNC panelists on TKI adjuvant therapy, that such therapy should not be recommended for routine use after nephrectomy for nmRCC (Level of evidence 1A). Nonetheless, such approach may represent an option in highly motivated patients. 


\section{References}

[1] Haas NB, Manola J, Uzzo RG, Flaherty KT, Wood CG, Kane C, et al. Adjuvant sunitinib or sorafenib for high-risk, non-metastatic renal-cell carcinoma (ECOG-ACRIN E2805): a double-blind, placebo-controlled, randomised, phase 3 trial. Lancet Lond Engl 2016;387:2008-16. doi:10.1016/S0140-6736(16)00559-6.

[2] Ravaud A, Motzer RJ, Pandha HS, George DJ, Pantuck AJ, Patel A, et al. Adjuvant Sunitinib in High-Risk Renal-Cell Carcinoma after Nephrectomy. N Engl J Med 2016;375:2246-54. doi:10.1056/NEJMoa1611406.

[3] Motzer RJ, Haas NB, Donskov F, Gross-Goupil M, Varlamov S, Kopyltsov E, et al. Randomized Phase III Trial of Adjuvant Pazopanib Versus Placebo After Nephrectomy in Patients With Localized or Locally Advanced Renal Cell Carcinoma. J Clin Oncol Off J Am Soc Clin Oncol 2017:JCO2017735324. doi:10.1200/JCO.2017.73.5324.

[4] KCRNC (Kidney Cancer Research Network of Canada) - Kidney Cancer Canada n.d. http://www.kidneycancercanada.ca/for-medical-professionals/kcrnc-kidney-cancer-researchnetwork-of-canada/ (accessed November 21, 2017).

[5] Canadian Kidney Cancer Forum 2017 - CUA Signups n.d. http://tech4pco.com/cua/videos/canadian-kidney-cancer-forum-2017 (accessed November 21, 2017).

[6] Meeting Library | MRC SORCE trial: Analysis of patients' presenting characteristics, tumor staging, and surgical approach. n.d. http://meetinglibrary.asco.org/record/91102/abstract (accessed October 12, 2017).

[7] Meeting Library | Everolimus (EVE) exposure as a predictor of toxicity (Tox) in renal cell cancer (RCC) patients (Pts) in the adjuvant setting: Results of a pharmacokinetic analysis for SWOG S0931 (EVEREST), a phase III study (NCT01120249). n.d. http://meetinglibrary.asco.org/record/144761/abstract (accessed October 12, 2017).

[8] Meeting Library | ATLAS study: A randomized double-blind phase 3 study of adjuvant axitinib versus placebo in subjects at high risk of recurrent renal cell carcinoma (RCC). n.d. http://meetinglibrary.asco.org/record/98459/abstract (accessed October 12, 2017).

[9] Chamie K, Donin NM, Klöpfer P, Bevan P, Fall B, Wilhelm O, et al. Adjuvant Weekly Girentuximab Following Nephrectomy for High-Risk Renal Cell Carcinoma: The ARISER Randomized Clinical Trial. JAMA Oncol 2017;3:913-20. doi:10.1001/jamaoncol.2016.4419. [10] Bandini M, Smith A, Zaffuto E, Pompe RS, Marchioni M, Capitanio U, et al. Effect of pathological high-risk features on cancer-specific mortality in non-metastatic clear cell renal cell carcinoma: a tool for optimizing patient selection for adjuvant therapy. World J Urol 2017. doi:10.1007/s00345-017-2093-6.

[11] Adelaiye R, Ciamporcero E, Miles KM, Sotomayor P, Bard J, Tsompana M, et al. Sunitinib dose escalation overcomes transient resistance in clear cell renal cell carcinoma and 
is associated with epigenetic modifications. Mol Cancer Ther 2015;14:513-22. doi:10.1158/1535-7163.MCT-14-0208.

[12] Raphael J, Thawer A, Bjarnason GA. Sunitinib dose-escalation after disease progression in metastatic renal cell carcinoma. Urol Oncol 2017. doi:10.1016/j.urolonc.2017.09.004.

[13] Gyawali B, Ando Y. Adjuvant sunitinib for high-risk-resected renal cell carcinoma: a meta-analysis of ASSURE and S-TRAC trials. Ann Oncol Off J Eur Soc Med Oncol 2017;28:898-9. doi:10.1093/annonc/mdw667.

[14] Bex A, Albiges L, Ljungberg B, Bensalah K, Dabestani S, Giles RH, et al. Updated European Association of Urology Guidelines Regarding Adjuvant Therapy for Renal Cell Carcinoma. Eur Urol 2017;71:719-22. doi:10.1016/j.eururo.2016.11.034. 


\section{Figures and Tables}

\section{Figure legends}

Fig. 1. Forest plot showing disease-free survival (DFS) for sunitinib, sorafenib and pazopanib 800mg, as recorded in ASSURE, S-TRAC and PROTECT trials. The left columns respectively show the study name, the active treatment agent and the sample size. The right column shows the hazard ratio (HR) and the 95\% confidence interval (95\% CI) for each study, as well as for their pooled effect.

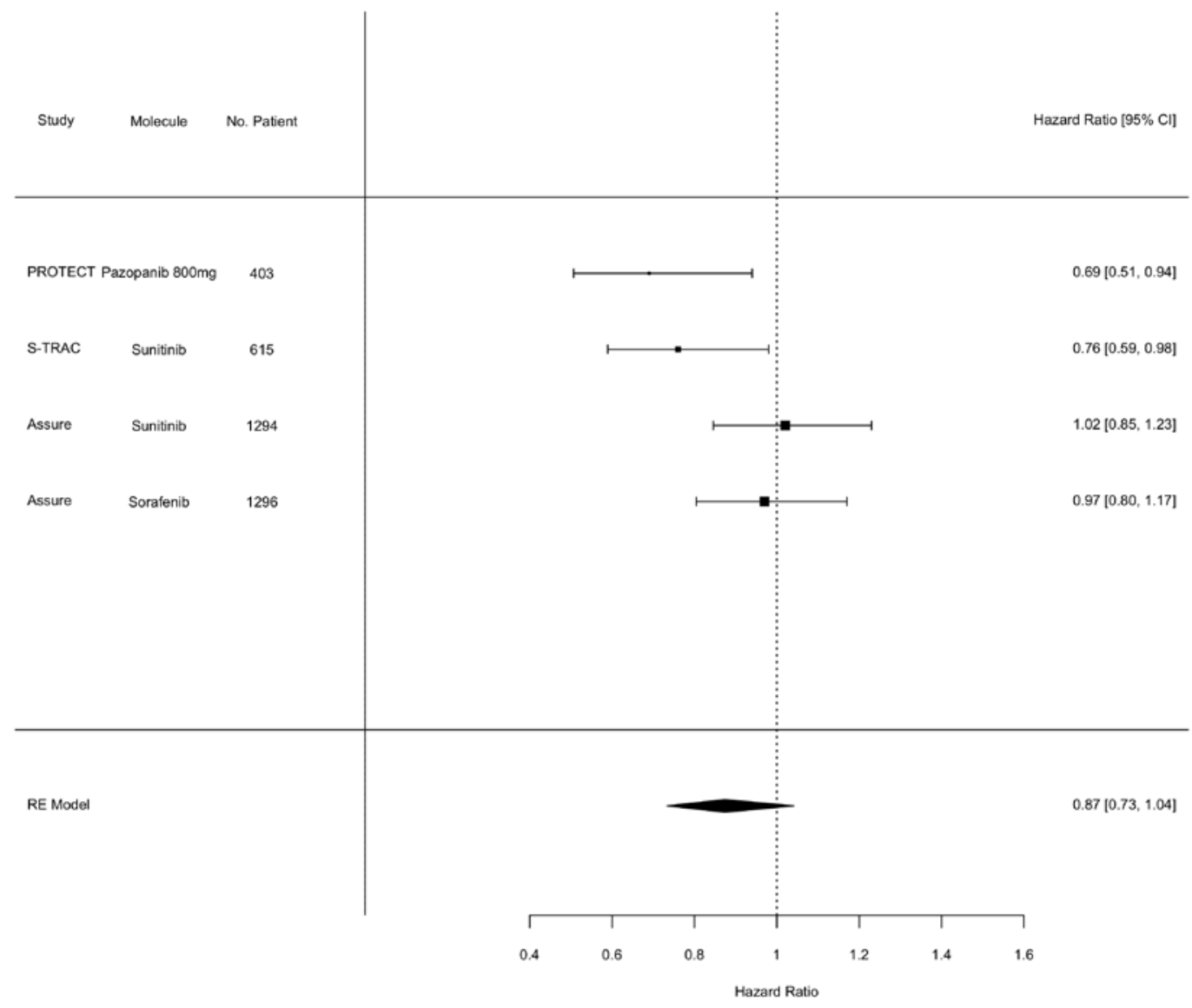


Fig. 2. Forest plot showing disease-free survival (DFS) for sunitinib, sorafenib and pazopanib 600mg, as recorded in ASSURE, S-TRAC and PROTECT trials. The left columns respectively show the study name, the active treatment agent and the sample size. The right column shows the hazard ratio (HR) and the 95\% confidence interval (95\% CI) for each study, as well as for their pooled effect.

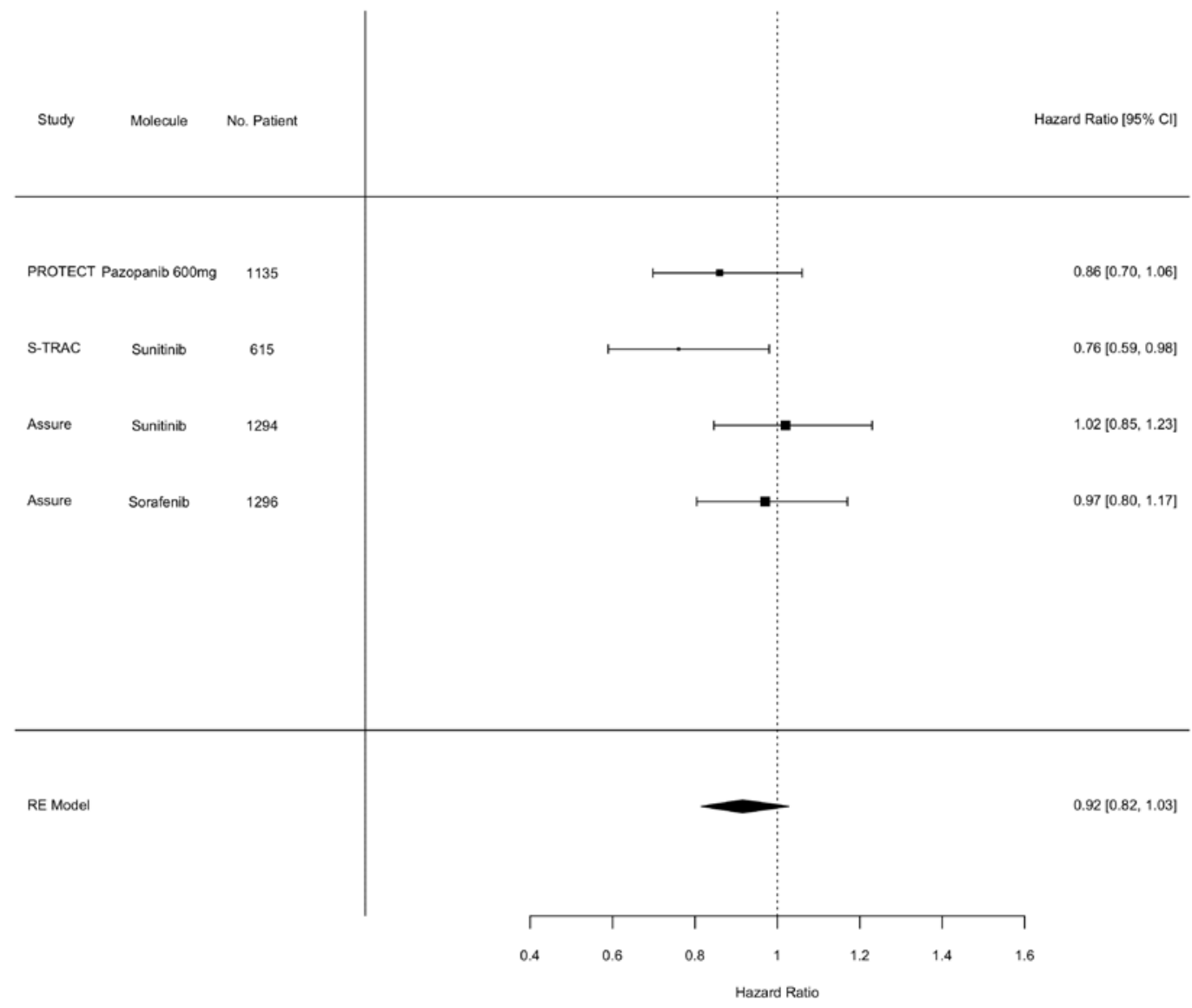


Fig. 3. Forest plot showing overall survival (OS) for sunitinib, sorafenib and pazopanib 800mg, as recorded in ASSURE, S-TRAC and PROTECT trials. The left columns respectively show the study name, the active treatment agent and the sample size. The right column shows the hazard ratio (HR) and the $95 \%$ confidence interval (95\% CI) for each study, as well as for their pooled effect.

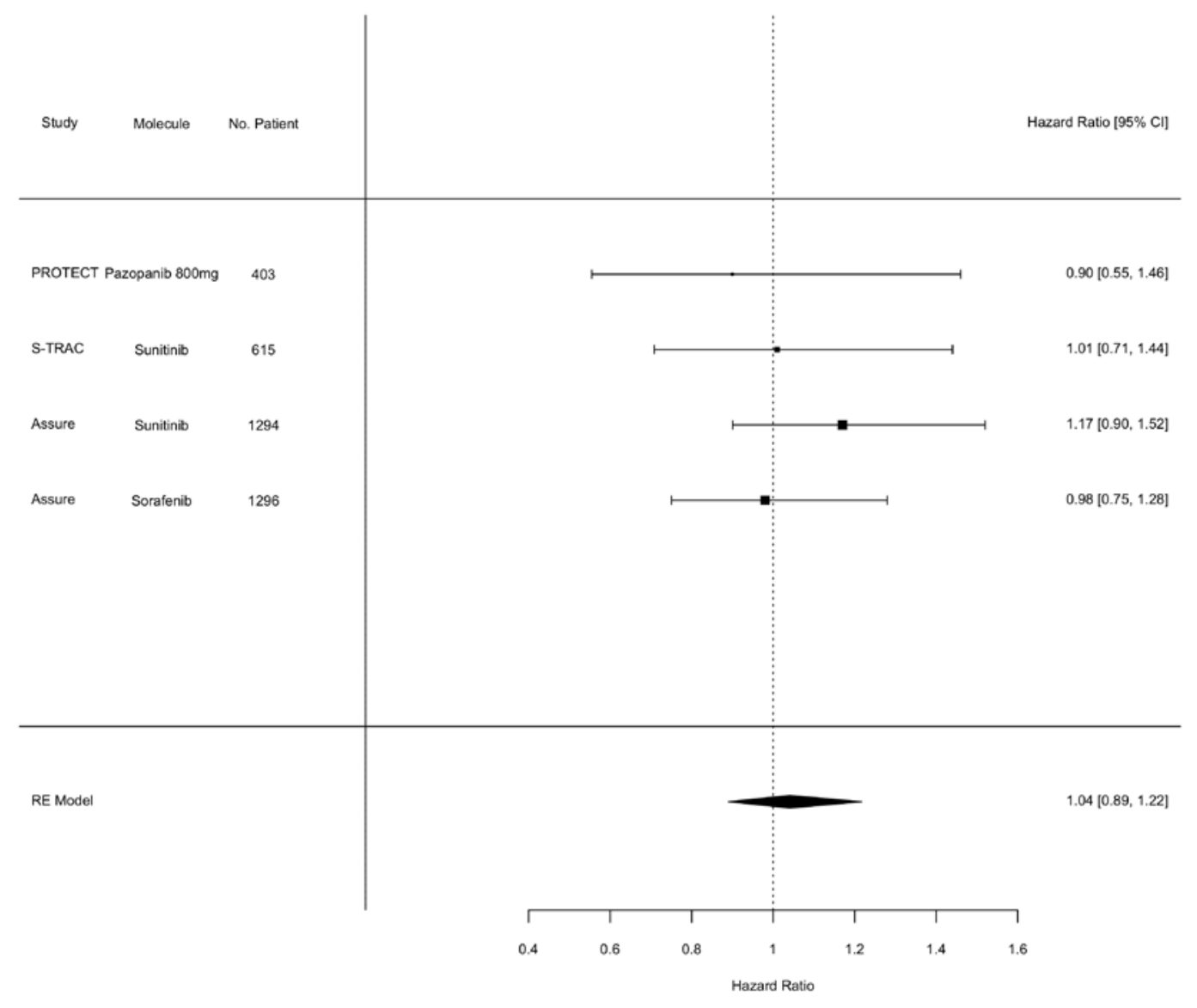


Fig. 4. Forest plot showing overall survival (OS) of sunitinib, sorafenib and pazopanib 600mg, as recorded in ASSURE, S-TRAC and PROTECT trials. The left columns respectively show the study name, the active treatment agent and the sample size. The right column shows the hazard ratio (HR) and the 95\% confidence interval (95\% CI) for each study, as well as for their pooled effect.

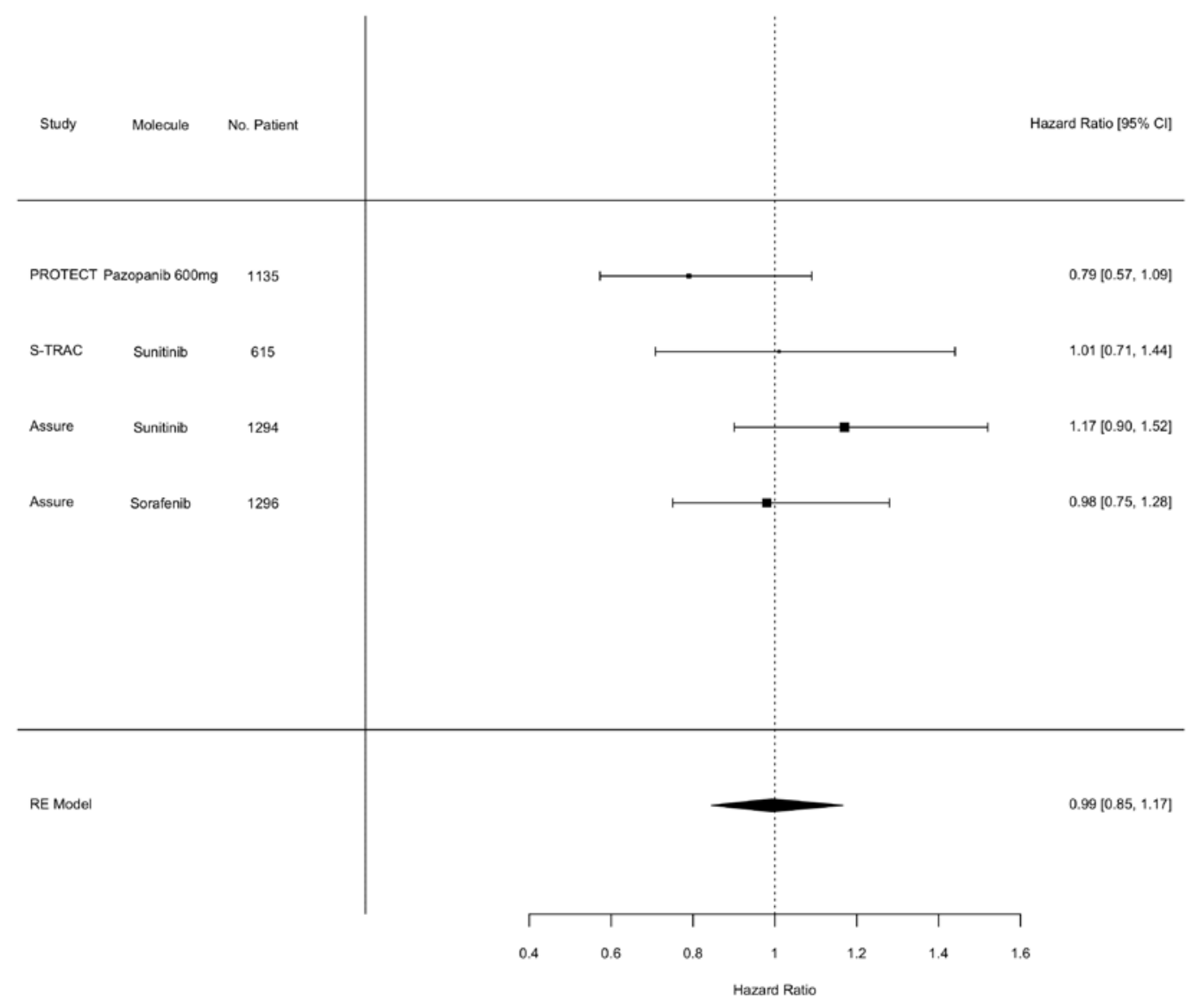


